\title{
ON STRONGLY NONLINEAR ELLIPTIC EQUATIONS WITH WEAK COERCITIVITY CONDITION
}

\author{
LÁSZLÓ SIMON
}

Abstract

We prove the existence and uniqueness of weak solutions of bound. ary value problems in an unbounded domain $\Omega \subset \mathbb{R}^{n}$ for strongly nonlinear $2 m$ order elliptic differential equations.

In this paper it will be proved existence and uniqueness of solutions of boundary value problems for the equation

$$
\begin{aligned}
\sum_{|\alpha|=m}(-1)^{m} D^{\alpha}\left[f_{\alpha}\left(x, D^{\alpha} u\right)\right]+ & \\
& +\sum_{|\alpha| \leqq m-1}(-1)^{|\alpha|} D^{\alpha}\left[g_{\alpha}\left(x, u, \ldots, D^{\beta} u, \ldots\right)\right]=F \text { in } \Omega
\end{aligned}
$$

where $\Omega$ is an unbounded domain in $\mathbb{R}^{n}, \alpha=\left(\alpha_{1}, \ldots, \alpha_{n}\right),|\alpha|=\sum_{j=1}^{n} \alpha_{j}$, $D_{j}=\frac{\partial}{\partial x_{j}}, D^{\alpha}=D_{1}^{\alpha_{1}} \ldots D_{n}^{\alpha_{n}},|\beta| \leqq m$.

Function $f_{\alpha}$ satisfies the Caratheodory conditions such that $\zeta_{\alpha} \mapsto$ $f_{\alpha}\left(x, \zeta_{\alpha}\right)$ is strictly monotone increasing, $f_{\alpha}(x, 0)=0$ and $f_{\alpha}, g_{\alpha}$ satisfy the "weak" coercitivity condition

$$
\sum_{|\alpha|=m} f_{\alpha}\left(x, \zeta_{\alpha}\right) \zeta_{\alpha}+\sum_{|\alpha| \leqq m-1} g_{\alpha}(x, \zeta) \zeta_{\alpha} \geqq c_{0} \sum_{|\alpha|=m}\left|\zeta_{\alpha}\right|^{p}
$$

with some constants $p>1, c_{0}>0$. Functions $g_{\alpha}$ have some polynomial growth in $D^{\beta} u$, but on $f_{\alpha}$ no growth restriction is imposed in $D^{\alpha} u$.

Similar result has been proved in [1] for the equation

$$
\sum_{|\alpha| \leqq m}(-1)^{|\alpha|} D^{\alpha}\left[g_{\alpha}\left(D^{\alpha} u\right)\right]=F
$$


in a bounded $\Omega$ if the condition

$$
g_{\alpha}\left(\zeta_{\alpha}\right) \zeta_{\alpha} \geqq c_{0}\left|\zeta_{\alpha}\right|^{p}-c_{1}, \quad|\alpha| \leqq m
$$

is fulfilled with some constants $p>1, c_{j}>0$. The proof of the existence theorem is based on a method called by F.E. Browdcr "clliptic superregularization" (see [1] - [3]). Our results can be extended to equations of the form

$$
\begin{aligned}
& \sum_{|\alpha|=m}(-1)^{m} D^{\alpha}\left[f_{\alpha}\left(x, u, \ldots, D^{\beta} u, \ldots\right]+\right. \\
& \quad+\sum_{|\alpha| \leq m-1}(-1)^{|\alpha|} D^{\alpha} \mid g_{\alpha}\left(x, u, \ldots, D^{\beta} u, \ldots\right]=F
\end{aligned}
$$

wherc $|\beta| \leqq m$ (see $[2]-[5])$.

It is to be mentioned that [ $[6]$ is connected with our result where $D$. Fortunato has considered cquation $L u+f(x, u)=0$; by $L$ is denoted a second order linear elliptic operator with weak coercitivity conditions in an unbounded domain. Similarly to our consideration, in $[6]$ the solution $u$ must satisfy the "asymptotic condition" $\int_{\Omega} \mid$ grad $\left.u\right|^{2} d x<+\infty$.

\section{The existence theorem}

Let $\Omega \subset \mathbb{R}^{n}$ be an unbounded domain with bounded boundary $\partial \Omega$, having the uniform $C^{n}$-regularity property and $\Omega_{r}=\Omega \cap B_{r}$ where $B_{r}=\left\{x \in \mathbb{R}^{n}:|x|<r\right\}$ (see [7]). Denote by $W_{p}^{m}(\Omega)$ the usual Sobolev space of real valued functions $u$ whose distributional derivatives belong to $L^{p}(\Omega)$. The norm on $W_{p}^{m}(\Omega)$ is

$$
\|u\|=\left\{\sum_{|\alpha| \leqq m} \int_{\Omega}\left|D^{\alpha} u\right|^{p} d x\right\}^{1 / p} .
$$

By $W_{p, \text { loc }}^{m}(\bar{\Omega})$ will be denoted the set of functions $f$ such that $\varphi f \in$ $W_{p}^{m}(\Omega)$ for all $\varphi \in C_{0}^{\infty}\left(\mathbb{R}^{n}\right)$, i.c. for all infinitely differentiable functions $\varphi$ with compact support.

Denote by $\tilde{W}_{p, 0}^{+n}(\Omega)$ the set of functions $u \in W_{p, \text { boc }}^{m_{b}}(\vec{\Omega})$ satisfying the conditions: $D^{\alpha} u \in L^{p}(\Omega)$ if $|\alpha|=m$ and the trace of $D^{\beta} u$ on $\partial \Omega$ equals to 0 if $|\beta| \leqq m-1$. The norm in $\tilde{W}_{p, 0}^{m}(\Omega)$ is defined by

$$
\|u\|_{\bar{W}_{r, 0}^{n}(\Omega)}=\left\{\sum_{|\alpha|=m} \int_{\Omega}\left|D^{\alpha} u\right|^{p} d x\right\}^{1 / p} .
$$


It is not difficult to show that $\tilde{W}_{p, 0}^{m}(\Omega)$ is a reflexive Banach space. Let $V$ be a closed linear subspace of $\tilde{W}_{p, 0}^{m}(\Omega)$.

Eet $N$ be the number of multiindices $\beta=\left(\beta_{1}, \ldots, \beta_{n}\right)$ satisfyirg $|\beta| \leqq$ $m$. Assume that

1. Functions $f_{\alpha}: \Omega \times \mathbb{R} \rightarrow \mathbb{R}(|\alpha|=m)$ satisfy the Caratheodory conditions, i.e. $f_{\alpha}\left(x, \zeta_{\alpha}\right)$ is measurable in $x$ for each fixed $\zeta_{\alpha} \in \mathbb{R}$ and it is continuous in $\zeta_{\alpha}$ for almost. all $x \in \Omega$.

II. $f_{\alpha}\left(x, \zeta_{\alpha}\right)$ is strictly ronotone increasing with respext to $\zeta_{\alpha x}$, $f_{\alpha}(x, 0)=0$.

III. For any $s>0$ there is a function $f_{\alpha, s}$ such that $f_{\alpha, s} \in L^{1}\left(\Omega_{r}\right)$ for each $r>0$ and

$$
\mid f_{\alpha}\left(x, \zeta_{\alpha} \mid \leqq f_{\alpha, s}(x) \text { if }\left|\zeta_{\alpha}\right| \leqq s .\right.
$$

Further, there exist constants $c_{1}, c_{2}>0$ and a function $f_{\alpha}^{*} \in L^{1}(\Omega)$ such that for a.t. $x \in \Omega$

$$
\left|f_{\alpha}^{*}\left(x, \zeta_{\alpha}\right)\right| \leqq f_{\alpha x}^{*}(x)+c_{1}\left|\zeta_{\alpha}\right|^{p-1} \text { if }\left|\zeta_{\alpha x}\right| \leqq c_{2}
$$

with some $p>1$.

IV. There exists a constart $c_{a}>0$ such that for all $\zeta_{\alpha} \in \mathbb{R}$, a.e $x \in \Omega$

$$
\left|f_{\alpha}\left(x, \zeta_{\alpha}\right)\right| \geqq c_{3}\left|\zeta_{\alpha}\right|^{p-1}
$$

V. Functions $g_{\alpha}: \Omega \times \mathbb{R}^{N} \rightarrow \mathbb{R}(|\alpha| \leqq m-1)$ satisfy the Caratheodory conditions.

VI. There exists a bounded domain $\Omega^{\prime} \subset \Omega$ such that $g_{\alpha}(x, \zeta)=0$ for all $\zeta \in \mathbb{R}^{N}$, a.c. $x \in \Omega \backslash \Omega^{\prime}$; further,

$$
\sum_{|\alpha| \leqq r^{\prime}-1} g_{\alpha}(x, \zeta) \zeta_{\alpha} \geqq 0
$$

VII. There exist constants $\rho_{|\alpha|}$, functions $\Phi_{\alpha} \in L^{p / \rho_{\alpha}}\left(\Omega^{\prime}\right)$ and a continuous function $C_{\alpha}$ such that.

$$
p-1 \leqq \rho_{|\alpha|}<p-1+\frac{(n-|\alpha|) p}{n}, \quad \rho_{|\alpha|} \leqq p
$$

and for all $\zeta \in \mathbb{R}^{N}$, a.e. $x \in \Omega^{\prime}$

$$
\left|g_{\alpha}(x, \zeta)\right| \leqq C_{\alpha}\left(\zeta^{\prime}\right)\left[\Phi_{\alpha}(x)+\left|\zeta^{\prime \prime}\right| P_{|a|}\right]
$$

where $\zeta=\left(\zeta^{\prime}, \zeta^{\prime \prime}\right)$ and $\zeta^{\prime}$ consists of those $\zeta_{\gamma}$ for which $|\gamma|<m-n / p$. 
Remark 1. Function $f_{\alpha}$ satisfies conditions I - IV e.g. in the following special case:

$$
f_{\alpha}\left(x, \zeta_{\alpha}\right)=\chi_{\alpha}(x) \varphi_{\alpha}\left(\zeta_{\alpha}\right)+\Psi_{\alpha}\left(\zeta_{\alpha}\right)
$$

where $\chi_{\alpha} \in L^{1}(\Omega), \chi_{\alpha} \geqq 0 ; \varphi_{\alpha}, \Psi_{\alpha}$ are continuous functions, $\varphi_{\alpha}$ is monotone increasing, $\Psi_{\alpha}$ is strictly monotone increasing, $\varphi_{\alpha}(0)=0$, $\Psi_{\alpha}(0)=0$ and

$$
c\left|\zeta_{\alpha x}\right|^{p-1} \leqq\left|\Psi_{\alpha}\left(\zeta_{\alpha}\right)\right|\left(\zeta_{\alpha} \in \mathbb{R}\right),\left|\Psi_{\alpha}\left(\zeta_{\alpha}\right)\right| \leqq \tilde{c}\left|\zeta_{\alpha}\right|^{p-1} \text { if }\left|\zeta_{\alpha}\right|<1
$$

by $c, \tilde{c}$ are deroted positive constants.

Theorem 1. Assume that conditions $I$ - VII are fulfilled. Then for any $G \in V^{*}$ (i.e. for linear continuous functional over $V$ ) with compact support there is $u \in V$ such that

$$
f_{\alpha}\left(x, D^{\alpha} u\right) D^{\alpha} u \in L^{1}(\Omega),
$$

$\left|f_{\alpha}\left(x, D^{\alpha} u\right)\right| \leqq f_{\alpha}^{(1)}+f_{\alpha}^{(2)}$ where $f_{\alpha}^{(1)} \in L^{1}(\Omega), f_{\alpha}^{(2)} \in L^{q}(\Omega), \frac{1}{p}+\frac{1}{q}=1$,

$$
\begin{aligned}
\sum_{|\alpha|=m} \int_{\Omega} f_{\alpha}\left(x, D^{\alpha} u\right) D^{\alpha} v d x+ & \\
& +\sum_{|\alpha| \leqq m-1} \int_{\Omega^{\prime}} g_{\alpha}\left(x, u, \ldots, D^{\beta} u, \ldots\right) D^{\alpha} v d x=\langle G, v\rangle
\end{aligned}
$$

for all $v \in C_{0}^{\infty}\left(\mathbb{R}^{n}\right)$ with $\left.v\right|_{\Omega} \in V$.

This theorem will be a simple consequence of Theorem 2 formulated below.

Let $V_{r}$ be the closure in $W_{p}^{m}\left(\Omega_{r}\right)$ of

$$
\left\{\left.\varphi\right|_{\Omega_{r}}: \varphi \in C_{0}^{\infty}\left(B_{r}\right) \cap V\right\}
$$

Then $V_{r}$ is a closed linear subspace of $W_{p}^{m}\left(\Omega_{r}\right)$ and -extending function $u \in V_{r}$ as 0 to $\Omega \backslash \Omega_{r^{-}}$the extensions belong to $V$. Let $s>\max \{n, p\}$ then by Sobolev's imbedding theorem $W_{s}^{m+1}\left(\Omega_{r}\right)$ is continuously and also compactly imbedded into $W_{p}^{m}\left(\Omega_{r}\right)$ and $C_{B}^{m}\left(\Omega_{r}\right)$ (see e.g. [7]) where $C_{B}^{m}\left(\Omega_{r}\right)$ denotes the set of $m$ times continuously differentiable functions 
$u$ with finite norm $\|u\|=\sum_{|\alpha| \leq m} \sup _{\Omega_{r}}\left|D^{\alpha} u\right|$. Denote by $\stackrel{\circ}{W}_{s}^{m+1}\left(\Omega_{r}\right)$ the closure in $W_{s}^{m+1}\left(\Omega_{r}\right)$ of

$$
\left\{\left.\varphi\right|_{\Omega_{r}}: \varphi \in C_{0}^{\infty}\left(B_{r}\right)\right\}
$$

Then -extending $u \in \stackrel{0}{W}_{s}^{m+1}\left(\Omega_{r}\right)$ as 0 to $\Omega \backslash \Omega_{\tau}-$ the extension belongs to $W_{s}^{m+1}(\Omega)$. Further, let

$$
W_{r}=\stackrel{\circ}{W}_{s}^{m+1}\left(\Omega_{r}\right) \cap V_{r}
$$

with the norm of $W_{s}^{m+1}\left(\Omega_{r}\right)$. Then $W_{r}$ is a closed linear subspace of $W_{s}^{m+1}\left(\Omega_{r}\right)$. Functions $u \in W_{r}$ will be extended to $\Omega \backslash \Omega_{r}$ as 0 .

For any $u, v \in W_{r}$ define

$$
\begin{aligned}
\left\langle S_{r}(u), v\right\rangle & =\sum_{|\alpha| \leqq m+1} \int_{\Omega_{r}}\left|D^{\alpha} u\right|^{s-2}\left(D^{\alpha} u\right)\left(D^{\alpha} v\right) d x \\
\left\langle T_{r}(u), v\right\rangle & =\sum_{|\alpha| \leqq m} \int_{\Omega_{r}} f_{\alpha}\left(x, D^{\alpha} u\right) D^{\alpha} v d x \\
\left\langle Q_{r}(u), v\right\rangle & =\sum_{|\alpha| \leqq m-1} \int_{\Omega^{\prime}} g_{\alpha}\left(x, u, \ldots, D^{\beta} u, \ldots\right) D^{\alpha} v d x
\end{aligned}
$$

By Hölder's inequality, Sobolev's imbedding theorem, assumptions I, III, V, VII $S_{r}, T_{r}, Q_{\tau}: W_{r} \rightarrow W_{r}^{*}$ are bounded nonlinear operators i.e. they map bounded sets of $W_{r}$ onto bounded sets of $W_{r}^{*}$.

Theorem 2. Assume that conditions I-VII are fulfilled, $G \subset V^{*}$ has compact support and $\lim _{l \rightarrow \infty} r_{l}=+\infty$. Then for sufficiently large l there exists at least one soluton $u_{l} \in W_{r_{l}}$ of

$$
\frac{1}{l}\left\langle S_{r_{i}}\left(u_{l}\right), v\right\rangle+\left\langle T_{r_{l}}\left(u_{l}\right), v\right\rangle+\left\langle Q_{r_{l}}\left(u_{l}\right), v\right\rangle=\langle G, v\rangle \text { for all } v \in W_{r_{i}}
$$

Further, there is a subsequence $\left(u_{l}^{\prime}\right)$ of $\left(u_{l}\right)$ which is weakly converging in $V$ to a function $u \in V$ satisfying (1.1) - (1.3). If (1.1) - (1.3) may have at most one solution then also $(u l)$ converges weakly to $u$.

Proof: Clearly, $\frac{1}{l} S_{r_{l}}$ is a pseudomonotone operator. Since $W_{r_{i}}$ is compactly imbedded into $C_{B}^{m}\left(\Omega_{r_{l}}\right)$ thus by use of assumptions I, III, V, VII 
it, is easy to show that also $\left(\frac{1}{i} S_{r_{t}}+T_{r_{l}}+Q_{r_{l}}\right): W_{r_{l}} \rightarrow W_{r_{t}}^{*}$ is pseudomonotone. Assumptions II, IV, VI imply that for each $u \in W_{v_{t}}$ (1.5)

$$
\left\langle\left(\frac{1}{l} S_{r_{l}}+T_{r_{t}}+Q_{r_{l}}\right)(u), u\right\rangle \geqq \frac{1}{l}\|u\|_{W_{r_{t}}}^{s}+c_{3} \sum_{|\alpha|=m} \int_{\Omega_{r_{l}}}\left|D^{\alpha} u\right|^{p} d x
$$

hence $\frac{1}{l} S_{r_{t}}+T_{r_{t}}+Q_{r_{t}}$ is cocrcive. So by the theory of pseudomonotone opcrators (see e.g. [8]) there is at least one solution $u_{l} \in W_{r_{l}}$ of (1.4).

Since $G$ has compact support (contained in $\Omega_{\dot{r}}$ ) thus

$$
\begin{aligned}
|\langle G, u\rangle| \leqq\|G\|_{V} \cdot\|u\|_{W_{p}^{m}\left(\Omega_{\tilde{r}}\right)} & \leqq c\|G\| v \cdot\left\{\sum_{|\alpha|=m} \int_{\Omega_{\bar{r}}}\left|D^{\alpha} u\right|^{p^{p}} d x\right\}^{1 / p} \leqq \\
& \leqq c\|G\| v \cdot\left\{\sum_{|\alpha|=m l} \int_{\Omega_{r_{p}}}\left|D^{\alpha} u\right|^{p} d x\right\}^{1 / p}
\end{aligned}
$$

for sufficiently large $l$. (The norm in $W_{p}^{m}\left(\Omega_{\tilde{r}}\right)$ is equivalent with $\left\{\sum_{|\alpha|=m \iota} \int_{\Omega_{\dot{r}}}\left|D^{\alpha} u\right|^{p r} d x\right\}^{1 / \rho}$ for functions satisfying $D^{\beta} u \mid r=0$ if $|\beta| \leqq$ $m-1$.)

From (1.4) - (1.6), $p>1$ it follows that

$$
\begin{gathered}
\frac{1}{l}\left\|u_{l}\right\|_{W_{\mathrm{r}_{t}}}^{s} \text { is bounded and } \\
\left\|u_{t}\right\|_{V} \text { is bounded. }
\end{gathered}
$$

Equality (1.4), VI and (1.8) imply that.

$$
\sum_{|\alpha| \leqq m} \int_{\Omega_{r_{i}}} f_{\alpha}\left(x, D^{\alpha} u_{l}\right) d x \text { is bounded. }
$$

By Hölder's inequality, for any fixed $j, v \in W_{r_{j}}$

$$
\left|\frac{1}{l}\left\langle S_{r_{l}}\left(u_{i}\right), v\right\rangle\right| \leqq \frac{1}{l}\left\|u_{t}\right\|_{W_{r_{t}}}^{s-1}\|v\| W_{r_{t}} \text { if } l \geqq j
$$

and so by (1.7)

$$
\lim _{l \rightarrow \infty} \frac{1}{l}\left\langle S_{r_{t}}(u l), v\right\rangle=0
$$


From (1.8) it follows that there are a subsequence $\left(u_{i}^{\prime}\right)$ of $\left(u_{i}\right)$ and $u \in V$ such that

$$
\left(u_{l}^{\prime}\right) \longrightarrow u \text { wcakly in } V
$$

and

$$
\left(D^{\gamma} u_{l}^{\prime}\right) \longrightarrow D^{\gamma} u \text { a.c. in } \Omega \text { for }|\gamma| \leqq m-1
$$

because by compact imbedding theorems it may be supposed that for any fixed $r>0$

$$
\left(D^{\gamma} u_{l}^{\prime}\right) \longrightarrow D^{\gamma} u \text { in } L^{p}\left(\Omega_{r}\right),|\gamma| \leqq m-1
$$

and by VII

$$
\left(D^{\gamma} u_{l}^{\prime}\right) \longrightarrow D^{\gamma} u \text { in } L^{q|\gamma|}\left(\Omega^{\prime}\right),|\gamma| \leqq m-1
$$

where $q_{|\gamma|}$ is defined by

$$
\frac{1}{p / \rho_{|\gamma|}}+\frac{1}{q_{|\gamma|}}=1
$$

Lemma 1. For oll $\alpha$ and each fixed $r>0$ the integrals

$$
\int_{\Omega_{r}}\left|f_{\alpha}\left(x, D^{\alpha} u_{i}^{\prime}\right)\right| d x
$$

are uniformly bounded and the functions $f_{\alpha}\left(x, D^{\alpha} u_{l}^{\prime}\right)$ are uniformly equiintegrable in $\Omega_{r}$.

Proof: From II it follows that for any $\zeta_{\alpha}, \tilde{\zeta}_{\alpha}$

$$
f_{\alpha \alpha}\left(x, \zeta_{\alpha}\right) \tilde{\zeta}_{\alpha} \leqq f_{\alpha}\left(x, \zeta_{\alpha}\right) \zeta_{\alpha}+f_{\alpha}\left(x, \vec{\zeta}_{\alpha}\right) \vec{\zeta}_{\alpha} \text {. }
$$

Applying this inequality to $\bar{\zeta}_{\alpha}=\rho \operatorname{sgn} f_{\alpha}\left(x, \zeta_{\alpha}\right)$ with arbitrary fixed number $\rho>0$ we obtain

$$
\rho\left[\operatorname{sgn} f_{\alpha}\left(x, \zeta_{\alpha}\right)\right] f_{\alpha}\left(x, \zeta_{\alpha}\right) \leqq f_{\alpha}\left(x, \zeta_{\alpha}\right) \zeta_{\alpha}+f_{\alpha}\left(x, \tilde{\zeta}_{\alpha}\right) \rho \operatorname{sgn} f_{\alpha}\left(x, \zeta_{\alpha}\right)
$$

where $\left|\tilde{\zeta}_{\alpha}\right|=\rho$. Thus by III we have

$$
\left|f_{\alpha}\left(x, \zeta_{\alpha x}\right)\right| \leqq \frac{f_{\alpha}\left(x, \zeta_{\alpha}\right) \zeta_{\alpha}}{\rho}+f_{\alpha, \rho}(x) \text {. }
$$

Combining this cstimation with (1.9) we obtain Lemma 1.

By using the methods of $[1],[2],[9]$ we obtain 
Lemma 2. There is a subsequence $\left(u_{l_{k}}\right)$ of $\left(u_{i}^{\prime}\right)$ such that

$$
\left(D^{\alpha} u_{t_{k}}\right) \longrightarrow D^{\alpha} u \text { a.e. in } \Omega \text { if }|\alpha|=m \text {. }
$$

(See [4, Lernma 4]).

Consider a fixed $v \in C_{0}^{\infty}\left(\mathbb{R}^{n}\right)$ such that $\left.v\right|_{\Omega} \in V$ and apply (1.4) to this $v$ and $l=l_{k}$. Then passing to the limit in (1.4), in virtue of I, V, (1.10) - (1.14), Lerrma 1, Lemma 2, Vitali's theorem and Hölder's inequality we obtain (1.3). (1.1) is a consequence of (1.9), II and Fatou's lemma. Since by III

$$
\begin{aligned}
\left|f_{\alpha}\left(x, \zeta_{\alpha}\right)\right| \leqq \sup _{\left|\zeta_{\alpha}\right| \leqq c_{2}}\left|f_{\alpha}\left(x, \zeta_{\alpha}\right)\right| & +\frac{1}{c_{2}}\left|f_{\alpha}\left(x, \zeta_{\alpha}\right) \zeta_{\alpha}\right| \leqq \\
& \leqq f_{\alpha}^{*}(x)+c_{1}\left|\zeta_{\alpha}\right|^{p-1}+\frac{1}{c_{2}}\left|f_{\alpha}\left(x, \zeta_{\alpha}\right) \zeta_{\alpha}\right|
\end{aligned}
$$

thus (1.1) implies (1.2).

\section{The uniqueness theorem}

In addtion to I - VII it will be assumed that the following conditions are fulfilled:

VIII. There is a constant $c_{A}$ such that for all $\zeta_{\alpha} \in \mathbb{R},|\alpha|=m$, a.e. $x \in \Omega$

$$
\left|f_{\alpha}\left(x, \zeta_{\alpha}\right)\right| \leqq c_{4} \mid f_{\alpha}\left(x,-\zeta_{\alpha} \mid .\right.
$$

IX. For each $\zeta, \bar{\zeta} \in \mathbb{R}^{N}$, a.e. $x \in \Omega$

$$
\sum_{|\alpha| \leqq m-1}\left[g_{\alpha}(x, \zeta)-g_{\alpha}(x, \bar{\zeta})\right]\left(\zeta_{\alpha}-\tilde{\zeta}_{\alpha}\right) \geqq 0 .
$$

IX. For each $\zeta, \tilde{\zeta} \in \mathbb{R}^{N}$, a.e. $x \in \Omega$

$$
\sum_{|\alpha| \leqq m-1}\left[g_{\alpha}(x, \zeta)-g_{\alpha}(x, \bar{\zeta})\right]\left(\zeta_{\alpha}-\bar{\zeta}_{\alpha}\right) \geqq 0
$$

X. $\Omega$ is a starlike domain in the following sense: there exist $x_{0} \in \mathbb{R}^{n}$ and $\delta>0$ such that $1<\lambda<1+\delta$ implies $\bar{\Omega}_{\lambda} \subset \Omega$ where

$$
\Omega_{\lambda}=\left\{x_{0}+\lambda\left(x-x_{0}\right): x \in \Omega\right\} \text {. }
$$

XI. There exist numbers $\varepsilon_{1}, \varepsilon_{2}, c_{5}>0$ and a function $k \in L^{q}(\Omega)$ such that for all $\zeta \in \mathbb{R}^{N}$, a.e. $x, x^{\prime} \in \Omega$

$$
\left|f_{\alpha}\left(x, \zeta_{\alpha}\right)\right| \leqq c_{5}\left|f_{\alpha}\left(x^{\prime}, \zeta_{\alpha}\right)\right|+k(x)
$$

if $\left|x-x^{\prime}\right| \leqq \hat{\epsilon}_{1}$ or if $x^{\prime}=x_{0}+\frac{1}{\lambda}\left(x-x_{01}\right)$ where $0<\lambda-1<\varepsilon_{2}, x_{0}$ is defined in $X$. 
Theorem 3. If conditions $I$ - XI are fulfiled then problem (1.1) (1.3) has a unique solution $u \in V$.

Remark 2. Functions $f_{\alpha}$ satisfy the conditions of Theorem 3 e.g. in the following special case:

$$
f_{\alpha}\left(x, \zeta_{\alpha}\right)=h_{\alpha}^{(1)}\left(\zeta_{\alpha}\right) \chi_{\alpha}(x)+h_{\alpha}^{(2)}\left(\zeta_{\alpha}\right)
$$

where $h_{\alpha}^{(j)}$ arc continuous, (for $j=2$ strictly) monotone increasing functions, $h_{\alpha}^{(j)}(0)=0$. Further, with suitable positive constants $c_{1}^{*}-c_{3}^{*}$ wc have

$$
\begin{aligned}
& \left|h_{\alpha}^{(j)}\left(-\zeta_{\alpha}\right)\right| \leqq c_{1}^{*}\left|h_{\alpha}^{(j)}\left(\zeta_{\alpha}\right)\right|, c_{2}^{*}\left|\zeta_{\alpha}\right|^{p-1} \leqq\left|h_{\alpha}^{(2)}\left(\zeta_{\alpha}\right)\right| ; \\
& \quad \text { for }\left|\zeta_{\alpha}\right|<1 \quad\left|h_{\alpha}^{(2)}\left(\zeta_{\alpha}\right)\right| \leqq c_{3}^{*}\left|\zeta_{\alpha}\right|^{p-1} .
\end{aligned}
$$

$\chi_{\alpha} \equiv 0$ or $\chi_{\alpha}>0, \chi_{\alpha} \in L^{1}(\Omega)$ and with some positive constants $\varepsilon_{1}, \varepsilon_{2}, c_{5}$

$\chi_{\alpha}(x) \leqq c_{5} \chi_{\alpha}\left(x^{\prime}\right)$ if $\left|x-x^{\prime}\right|<\varepsilon_{1}$ or $x^{\prime}=x_{0}+\frac{1}{\lambda}\left(x-x_{0}\right)$ where $0<\lambda-1<\varepsilon_{2} . \chi_{\alpha}$ satisfies the above conditions e.g. if $x_{11}=0, \chi_{\alpha}$ is continuous, positive and out of some $B_{a} \quad \chi_{\alpha}(x)=\chi_{\alpha}^{1}(|x|)$ where $\chi_{x x}^{1}$ is monotone decreasing and its derivative is bounded.

In the proof of Theorem 3 we need

Lemma 3. For each $\zeta_{\alpha}, \tilde{\zeta}_{\alpha}$, a.e. $x \in \Omega$

$$
\left|f_{\alpha}\left(x, \zeta_{\alpha}\right) \tilde{\zeta}_{\alpha x}\right| \leqq c_{1}\left[f_{\alpha}\left(x, \zeta_{\alpha}\right) \zeta_{\alpha}+f_{\alpha}\left(x, \tilde{\zeta}_{\alpha x}\right) \zeta_{\alpha x}\right]
$$

Proof: Defire $\tilde{\zeta}_{\alpha}^{\prime}=\left|\tilde{\zeta}_{\alpha}\right|\left(\operatorname{sgn} \zeta_{\alpha}\right)$ then II implies

$$
f_{\alpha}\left(x, \zeta_{\alpha}\right) \tilde{\zeta}_{\alpha}^{\prime}+f_{\alpha}\left(x, \tilde{\zeta}_{\alpha}^{\prime}\right) \zeta_{\alpha} \leqq f_{\alpha}\left(x, \zeta_{\alpha}\right) \zeta_{\alpha}+f_{c x}\left(x, \tilde{\zeta}_{\alpha}^{\prime}\right) \dot{\zeta}_{\alpha}^{\prime}
$$

whence by $f_{\alpha}\left(x, \zeta_{\alpha}\right) \zeta_{\alpha} \geqq 0, f_{\alpha}\left(x, \bar{\zeta}_{\alpha}^{\prime}\right) \bar{\zeta}_{\alpha}^{\prime} \geqq 0$

$$
f_{\alpha}\left(x, \zeta_{\alpha}\right) \tilde{\zeta}_{\alpha}^{\prime} \leqq f_{\alpha}\left(x, \zeta_{\alpha}\right) \zeta_{\alpha}+\dot{f}_{\alpha}\left(x, \tilde{\zeta}_{\alpha}^{\prime}\right) \tilde{\zeta}_{\alpha}^{\prime}
$$

Thus in virtue of $f_{\alpha}\left(x, \zeta_{\alpha}\right) \zeta_{\alpha} \geqq 0$, VIII we have

$$
\begin{aligned}
& \left|f_{\alpha}\left(x, \zeta_{\alpha}\right) \vec{\zeta}_{\alpha}\right|=f_{\alpha}\left(x, \zeta_{\alpha}\right) \tilde{\zeta}_{\alpha}^{\prime} \leqq \\
& \quad \leqq f_{\alpha}\left(x, \zeta_{\alpha}\right) \zeta_{\alpha}+f_{\alpha}\left(x, \tilde{\zeta}_{\alpha}^{\prime}\right) \tilde{\zeta}_{\alpha}^{\prime} \leqq f_{\alpha}\left(x, \zeta_{\alpha}\right) \zeta_{\alpha}+c_{\alpha} f_{\alpha}\left(x, \tilde{\zeta}_{\alpha}\right) \vec{\zeta}_{\alpha}
\end{aligned}
$$


The Proof of Theorcm 3: Assume that $u=u^{\prime}$ and $u=u^{\prime \prime}$ satisfy (1.1) - (1.3). We shall show that (1.3) is fulfilled with $v=u^{\prime}, v=u^{\prime \prime}$. This will imply $u^{\prime}=u^{\prime \prime}$ a.e. since then

$$
\begin{aligned}
& \sum_{|\alpha|-m_{k}} \int_{\Omega}\left[f_{x \alpha}\left(x, D^{\alpha x} u^{\prime}\right)-f_{\alpha}\left(x, D^{\alpha} u^{\prime \prime}\right)\right]\left(D^{\alpha} u^{\prime}-D^{\alpha} u^{\prime \prime}\right) d x+ \\
&+ \sum_{\left[\alpha \mid \leqq m_{-1}-1\right.} \int_{\Omega^{\prime}}\left[g_{\alpha}\left(x, u^{\prime}, \ldots, D^{\beta} u^{\prime}, \ldots\right)-g_{\alpha}\left(x, u^{\prime \prime}, \ldots, D^{\beta} u^{\prime \prime}, \ldots\right)\right] \\
&\left(D^{\alpha} u^{\prime}-D^{\alpha} u^{\prime \prime}\right) d x=0
\end{aligned}
$$

and so by II, IX $D^{\alpha} u u^{\prime}=D^{\alpha} u^{\prime \prime}$ a.e. in $\Omega$ if $|\alpha|=m$ which implies $u^{\prime}=u^{\prime \prime}$ a.e. as $u^{\prime}, u^{\prime \prime} \in \tilde{W}_{p, t}^{m+1}(\Omega)$.

Let $\lambda_{j}$ be a sequence of numbers such that $\operatorname{limr}\left(\lambda_{j}\right)=1$ and $1-\delta<$ $\lambda_{j}<1, \quad j=1,2, \ldots$. Define functions $v_{j}$ in $\mathbb{R}^{r_{2}}$ by

$$
v_{j}(x)=\left\{\begin{array}{l}
u^{\prime}\left(x_{0}+\frac{1}{\lambda_{j}}\left(x-x_{0}\right)\right) \text { if } x \in \Omega_{\lambda_{j}} \\
0 \text { othcrwise }
\end{array}\right.
$$

and consider the convolution $v_{j} * \eta_{\varepsilon}$ where $\varepsilon$ is a positive number and $\eta_{\varepsilon} \in C_{0}^{\infty}\left(\mathbb{P}^{n}\right)$ is such that $\eta_{\varepsilon} \geqq 0, \eta_{\varepsilon}(x)=0$ for $|x|>\varepsilon$ and $\int \eta_{\varepsilon} d x=1$. Then $v_{j} * \eta_{E} \in C^{\infty}\left(\mathbb{R}^{n}\right)$ and by Hölder's inequality for $|\alpha|=m$

$$
D^{\alpha}\left(v_{j} * \eta_{\varepsilon}\right)=D^{\alpha} v_{j} * \eta_{\varepsilon} \in L^{p}\left(\mathbb{R}^{n}\right) \cap L^{\infty}\left(\mathbb{R}^{n}\right)
$$

since the trace of $D^{\beta} v_{j}$ on $\partial \Omega_{\lambda_{j}}$ is 0 if $|\beta| \leqq m-1$.

By using an idea of $\mathrm{V}$. Komornik, we show that. (1.3) holds with $u=$ $u^{\prime \prime}, v=v_{j} * \eta_{\varepsilon}$ if $\varepsilon>0$ is sufficiently small.

Let $w=v_{j} * \eta_{k}$. Further, consider a fixed function $\varphi \in C_{0}^{\infty}\left(\mathbb{F}^{n}\right)$ such that $0 \leqq \varphi \leqq 1, \varphi(x)=0$ if $|x| \geqq 1, \varphi(x)=1$ if $|x| \leqq 1 / 2$ and define $w_{k}$ by

$$
w_{k}(x)=\varphi\left(\frac{x}{k}\right) w(x)
$$

Then

$$
D^{\alpha x} u_{k}(x)=\sum_{\gamma \leqq \alpha} c_{\gamma} \frac{1}{k|\gamma|} D^{\gamma} \varphi\left(\frac{x}{k}\right) D^{\alpha-\gamma} w(x)
$$

whence: 


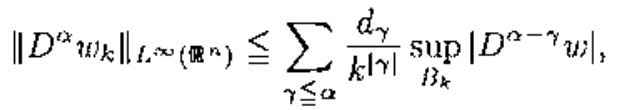

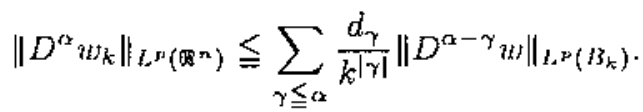

In order to estimate the right hand sides of $(2.4),(2.5)$ we prove estimations

$$
\begin{aligned}
& \|f\|_{L, \infty}\left(B_{k}\right) \\
& \| \text { const } k^{l} \sum_{|\beta|=l}\left\|D^{\beta} f\right\|_{L^{\infty}\left(B_{k}\right)}, \\
& \|f\|_{L, p\left(B_{k}\right)} \leqq \text { cornst. } k^{l} \sum_{|\beta|=l}\left\|D^{\beta} f\right\|_{l^{n}\left(l B_{k}\right)},
\end{aligned}
$$

if $f^{\prime}(x)=0$ in a noighbourhood of 0 . Indexd, we have

$$
f(x)=\int_{0}^{|x|} \frac{x}{|x|} D f\left(t \frac{x}{|x|}\right) d t
$$

and so

$$
\|f\|_{l \infty}\left(B_{k}\right) \leqq k\|D f\|_{l \infty\left(B_{k}\right)} .
$$

Further,

$$
|f(x)| \leqq \int_{0}^{|x|}\left|D f\left(t \frac{x}{|x|}\right)\right| d t \leqq|x|^{\frac{1}{2}}\left(\int_{0}^{|x|}\left|D f\left(t \frac{x}{|x|}\right)^{p}\right| d t\right)^{1 / p}
$$

and, consequently, by using the notation $S_{r}=\left\{x \in \mathbb{P}^{r}:|x|=r\right\}$

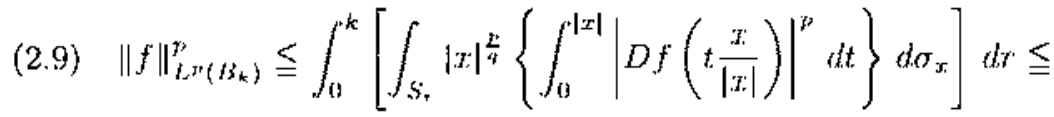

$$
\begin{aligned}
& \leqq \int_{0}^{k} r^{\frac{\mu}{q}} d r\|D f\|_{L,\left(B_{k}\right)}^{p}=\frac{1}{p} k^{\frac{p}{q}+1}\|D f\|_{L^{\prime}\left(B_{k}\right)}^{p^{\prime}},\|f\|_{L^{\mu}\left(B_{k}\right)} \leqq \\
& \leqq\left(\frac{1}{p}\right)^{\frac{1}{p}} k\|D f\|_{L, r\left(B_{k}\right)}
\end{aligned}
$$

Applying (2.8) resp. (2.9) suecessively we obtain (2.6) resp. (2.7).

Clearly, without loss of generality, we may ixsume that $0 \in \partial \Omega$ and so for sufficiently small $\varepsilon>0 \quad w=v_{j} * \eta_{\epsilon}$ is $0 \mathrm{in}$ a neighbourhood of 0 . 
Thus we may estimate the right hand sides of (2.4), (2.5) by (2.6) resp. (2.7) and so (2.2) implies that

$$
\left\|D^{\alpha} w_{k}\right\|_{L^{\infty}\left(\mathbb{k}^{n}\right)},\left\|D^{\alpha} w_{k}\right\|_{L^{r}\left(\mathbb{R}^{n}\right)}
$$

are bounded $k=1,2, \ldots$. Further, by the definition of $w_{k}$

$$
w_{k}=w \text { in } B_{\frac{k}{2}}
$$

Therefore, applying (1.3) to $u=u^{\prime \prime}, v=w_{k}$, by using Vitali's theorem we obtain as $k \rightarrow \infty$ that (1.3) holds with $u=u^{\prime \prime}, v=v_{j} * \eta_{c}$.

Now, we shall prove that (1.3) is valid also with $u=u^{\prime \prime}, v=v_{j}$. Let $\varepsilon_{k}>0$ be such that $\lim \left(\varepsilon_{k}\right)=0$. Then for each fixed $r \geqq r_{0}$

$$
\lim _{k \rightarrow \infty}\left\|v_{j} * \eta_{\varepsilon_{k}}-v_{k}\right\|_{W_{p}^{m}}\left(\Omega_{r}\right)=0
$$

(see e.g. [7]), consequertily, for a suitable subsequence $\left(\varepsilon_{k}^{\prime}\right)$ of $\left(\varepsilon_{k}\right)$

$$
D^{\alpha}\left(v_{j} * \eta_{e_{k}^{\prime}}\right) \longrightarrow D^{\alpha} v_{j} \quad(|\alpha| \leqq n)
$$

a.e. in $\Omega_{r}$. Applying this statement to $r=r_{0}, r_{0}+1, r_{0}+2, \ldots$ we may extract a subscquence $\left(\varepsilon_{k}^{\prime \prime}\right)$ such that $(2.10)$ holds a.e. in $\Omega$.

Now we prove that for a fixed $j,|\alpha|=m$ the sequence of functions

$$
f_{\alpha}\left(x, D^{\alpha} u^{\prime \prime}\right) D^{\alpha}\left(v_{j} * \eta_{e_{k}^{\prime \prime}}\right), \quad k=1,2, \ldots
$$

is equintegrable in $\Omega$. According to (2.1) $v_{j}(y)=u^{\prime}\left(\Phi_{j}(y)\right)$ where $\Phi_{j}(y)=x_{0}+\frac{1}{\lambda_{j}}\left(y-x_{0}\right)$ (out of $\Omega \quad u^{\prime}$ is considered to be 0$)$. Conscquently, with some positive constant $e_{0}>0$ we obtain

$$
\begin{aligned}
\left|D^{\alpha}\left(v_{j} * \eta_{\varepsilon_{k}^{\prime \prime}}\right)(x)\right|=\left|\int_{\mathbb{R}^{n}} D^{\alpha} y_{j}(y) \eta_{\varepsilon_{k^{\prime \prime}}}(x-y) d y\right| & \leqq \\
& \leqq c_{6} \int_{\mathbb{R}^{n}}\left|D^{\alpha} u^{\prime}\left(\Phi_{j}(y)\right)\right| \eta_{\varepsilon_{k}^{\prime \prime}}(x-y) d y .
\end{aligned}
$$

Therefore, by using Lemma 3, XI and $\int_{\mathbb{R}^{n}} \eta_{\varepsilon_{x}^{\prime \prime}}=1$, functions $(2.11)$ can 
be estimated for sufficiently large $k$ in the following way:

$$
\begin{aligned}
& \left|f_{\alpha}\left(x, D^{\alpha} u^{\prime \prime}(x)\right) D^{\alpha}\left(v_{j} * \eta_{\varepsilon_{x}^{\prime \prime}}\right)(x)\right| \leqq \\
& \leqq c_{6} \int_{\mathbb{R}^{n}}\left|f_{\alpha}\left(x, D^{\alpha} u^{\prime \prime}(x)\right) D^{\alpha} u^{\prime}\left(\Phi_{j}(y)\right)\right| \eta_{\varepsilon_{k}^{\prime \prime}}(x-y) d y \leqq \\
& \leqq c_{4} c_{6} \int_{\mathbb{R}^{n}} f_{\alpha}\left(x, D^{\alpha} u^{\prime \prime}(x)\right) D^{\alpha} u^{\prime \prime}(x) \eta_{e_{x}^{\prime \prime}}(x-y) d y+ \\
& +c_{4} c_{5} \int_{\mathbb{Q}^{n}} f_{\alpha}\left(x, D^{\alpha} u^{\prime}\left(\Phi_{j}(y)\right)\right) D^{\alpha} u^{\prime}\left(\Phi_{j}(y)\right) \eta_{\varepsilon_{k}^{\prime \prime}}(x-y) d y \leqq \\
& \leqq c_{4} c_{6} f_{\alpha}\left(x, D^{\alpha} u^{\prime \prime}(x)\right) D^{\alpha} u^{\prime \prime}(x)+ \\
& +\epsilon_{4} c_{5}^{2} c_{6} \int_{\mathbb{R}^{n}} f_{\alpha}\left(\Phi_{j}(y), D^{\alpha} u^{\prime}\left(\Phi_{j}(y)\right)\right) D^{\alpha} u^{\prime}\left(\Phi_{j}(y)\right) \eta_{\varepsilon_{k}^{\prime \prime}}(x-y) d y+ \\
& +2 c_{4} c_{6} k(x) \int_{\mathbb{R}^{n}}\left|D^{\alpha} u^{\prime}\left(\Phi_{j}(y)\right)\right| \eta_{\epsilon_{k}^{\prime \prime}}(x-y) d y .
\end{aligned}
$$

In the last sum the first term is Lebesgue integrable in $\Omega$, the second and third terms are equiintegrable in $\Omega(k=1,2, \ldots)$ since for some $\Omega_{0} \supset \bar{\Omega}$

$$
\begin{aligned}
y \mapsto f_{\alpha}\left(\Phi_{j}(y), D^{\alpha} u^{\prime}\left(\Phi_{j}(y)\right) D^{\alpha} u^{\prime}\left(\Phi_{j}(y)\right) \in L^{1}\left(\Omega_{0}\right),\right. \\
D^{\alpha} u^{\prime}\left(\Phi_{j}(y)\right) \in L^{p}\left(\Omega_{0}\right), k \in L^{q}(\Omega) .
\end{aligned}
$$

Thus the sequence of functions (2.11) is equiintegrable in $\Omega$ and so by (2.10) and Vitali's theorem we find

$$
\lim _{k \rightarrow \infty} \int_{\Omega} f_{\alpha}\left(x, D^{\alpha} u^{\prime \prime}\right) D^{\alpha}\left(v_{j} * \eta_{\varepsilon_{k}^{\prime \prime}}\right) d x=\int_{\Omega} f_{\alpha}\left(x, D^{\alpha} u^{\prime \prime}\right) D^{\alpha} v_{j} d x
$$

By using (2.10), VI, VII, Sobolev's imbedding theorem, Hölder's inequality and Vitali's theorem it is not difficult to show that for $|\alpha| \leqq m-1$

$$
\begin{aligned}
\lim _{k \rightarrow \infty} \int_{\Omega^{\prime}} g_{\alpha}\left(x, u^{\prime \prime}, \ldots,\right. & \left.D^{\beta} u^{\prime \prime}, \ldots\right) D^{\alpha}\left(v_{j} * \eta_{\varepsilon_{k}^{\prime \prime}}\right) d x= \\
& =\int_{\Omega^{\prime}} g_{\alpha}\left(x, u^{\prime \prime}, \ldots, D^{\beta} u^{\prime \prime}, \ldots\right) D^{\prime \alpha} v_{j} d x .
\end{aligned}
$$

Finally, $\left\|v_{j} * \eta_{\varepsilon_{k}^{\prime \prime}}\right\|_{V} \leqq\left\|v_{j}\right\|_{V}$, thus it may be supposed: we have chosen subsequence $\left(\varepsilon_{k}^{\prime \prime}\right)$ of $\left(\varepsilon_{k}^{\prime}\right)$ such that

$$
\left(v_{j} * \eta_{\varepsilon_{k}^{\prime \prime}}\right) \longrightarrow v_{j} \text { weakly in } V .
$$

Since (1.3) holds with $u=u^{\prime \prime}, v=v_{j} * \eta_{\varepsilon_{k}^{\prime \prime}}$, thus from (2.12) - (2.14) we obtain as $k \rightarrow \infty$ that (1.3) holds with $u=u^{\prime \prime}, v=v_{j}$. Consequently, similarly to the above arguments, we obtain as $j \rightarrow \infty$ that (1.3) is valid for $u=u^{\prime \prime}, v=u^{\prime}$. Analogously can be considered cases $u=u^{\prime \prime}, v=$ $u^{\prime \prime} ; u=u^{\prime}, v=u^{\prime}$ resp. $u^{\prime \prime}$. 


\section{References}

1. Mustone., V., Simader, C.G., On the existence and uniqueness of solutions for strongly nonlinear elliptic variational problems, $A n n$. Acad. Sci. Fenn. Ser. A I. Math. 6 (1981), 233-254.

2. LANDES, R., Quasilincar elliptic operators and weak solutions of the Euler equation, Manuscripta Math. 27 (1979), 47-72.

3. SimADER, C.G., Remarks on uniqueness and stability of weak solutions of strongly nonlinear elliptic cquations, Bayreuther Mathematische Schriften 11 (1982), 67-79.

4. SIMON, L., On strongly nonlinear elliptic variational inequalities, Acta. Math. Acad. Sci. Hung. 52 (1988), 147-164.

5. Simon, L., (n uniqueness, regularity and stability of solutions of strongly nonlinear clliptic variational incqualities, Acta Math. Acad. Sci. Hung. 55 (1990), 379--392.

6. Fontensio, D., Remarks on some nonlinear boundary valuc problerns, Annali di Matematica Pura Appl. (4) 124 (1980), 217-231.

7. Adnмs, R., "Sobolev spaces," Academic Press, New York, San Francisco, London, 1975.

8. LiONs, J.L., "Quelques méthodes de résolution des problèmes aux limites non linécaires:" Dunod, Gauthicr-Villars, Paris, 1969.

3. LANbEs, R, On Galerkin's method in the existence thcory of quasilinear elliptic equations, J. Funetional Anulysis 39 (1980), 123-148.

Eöł.vös Loránd Thudományegyetem

1088 Budapesi

Murcum krt. 6-8

IIUNGARY

Retut el 29 d'Abril de 1991 